\title{
First Measurement of Electron Temperature and Density Profiles for Spherical Tokamak Plasmas Sustained by Lower Hybrid Waves
}

\author{
Hiro TOGASHI, Akira EJIRI, Hiroto HOMMA, Takahiro SHINYA, Yuichi TAKASE, \\ Kazuya TOIDA, Naoto TSUJII, Takashi YAMAGUCHI, Yusuke YOSHIDA, \\ Makoto HASEGAWA $^{1)}$, Yoshihiko NAGASHIMA ${ }^{1)}$, Hirokazu FURUI, Kenta NAKAMURA, \\ Wataru TAKAHASHI, Toshihiro TAKEUCHI, Masateru SONEHARA, \\ Satoru YAJIMA and Hibiki YAMAZAKI \\ The University of Tokyo, Kashiwa 277-8561, Japan \\ ${ }^{1)}$ Kyushu University, Kasuga 816-8580, Japan
}

(Received 29 June 2015 / Accepted 1 September 2015)

\begin{abstract}
In this research, the profiles of electron temperature $T_{\mathrm{e}}$ and density $n_{\mathrm{e}}$ for a spherical tokamak with the plasma current sustained by lower hybrid waves alone have been measured for the first time using Thomson scattering diagnostics in the TST- 2 spherical tokamak device. The $T_{\mathrm{e}}$ profile was hollow and the $n_{\mathrm{e}}$ profile was like bell-shaped. $T_{\mathrm{e}}$ and $n_{\mathrm{e}}$ near the plasma center were $6 \mathrm{eV}$ and $6 \times 10^{17} \mathrm{~m}^{-3}$, respectively, leading to a pressure of $0.6 \mathrm{~Pa}$. On the other hand, whole pressure at the plasma center calculated using an equilibrium reconstruction code EFIT was around $20 \mathrm{~Pa}$. Therefore, it is suggested that fast electrons play an important role in the plasma equilibrium.
\end{abstract}

(c) 2015 The Japan Society of Plasma Science and Nuclear Fusion Research

Keywords: TST-2, spherical tokamak, Thomson scattering, lower hybrid wave, current drive

DOI: $10.1585 /$ pfr.10.1202082

In order to realize a compact spherical tokamak (ST) reactor, which has no space for a central solenoid, a plasma current needs to be started-up non-inductively. Plasma current start-up experiments using electron cyclotron wave (ECW) have been performed in several ST devices [1-4]. On the other hand, the lower hybrid wave (LHW) is used in the TST-2 [5]. A successful lower hybrid current drive (LHCD) was achieved with a high efficiency in many conventional tokamaks, but the efficiency is low for ST devices $[6,7]$. For LHCD optimization in STs, it is important to obtain electron temperature $T_{\mathrm{e}}$ and density $n_{\mathrm{e}}$ profiles, leading to understanding of wave propagation and absorption. $T_{\mathrm{e}}$ and $n_{\mathrm{e}}$ for plasmas sustained by the LHW were measured in Globus-M [7], but the profiles were not reported. Thomson scattering (TS) diagnostics is widely used to measure the profiles. However, TS measurement becomes difficult when electron density is low because of the weak TS signal. Plasmas started-up by radiofrequency (RF) generally have lower density than those for ohmically started-up plasmas by tens to hundreds times. In order to improve the signal-to-noise ratio in such lowdensity plasmas, several techniques have been adopted, including averaging many raw TS signals over reproducible discharges [8], and a multi-pass scheme wherein a laser pulse makes many round trips in an optical cavity $[9,10]$.

TST-2 is a spherical tokamak with major radius $R=$ $0.36 \mathrm{~m}$ and minor radius $a=0.23 \mathrm{~m}$. Typical discharges in the present experiment are shown in Fig. 1. A $2 \mathrm{~kW}$ $\mathrm{ECW}$ is injected for plasma initiation, and the plasma current is started-up and sustained by a LHW power of $20 \mathrm{~kW}$. The plasma current $I_{\mathrm{p}}$ is approximately $5 \mathrm{kA}$, and the line-

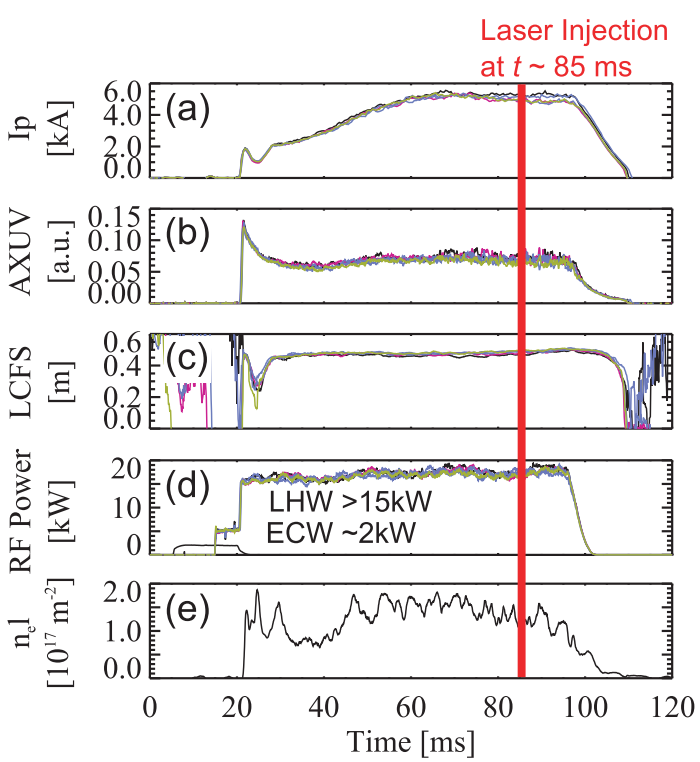

Fig. 1 Waveforms of (a) plasma currents, (b) radiation measured by an absolute extreme ultraviolet (AXUV) detector, (c) LCFSs, and (d) LHW and ECW injected powers for 5 discharges. Part (f) shows a typical line-integrated density at the midplane. 

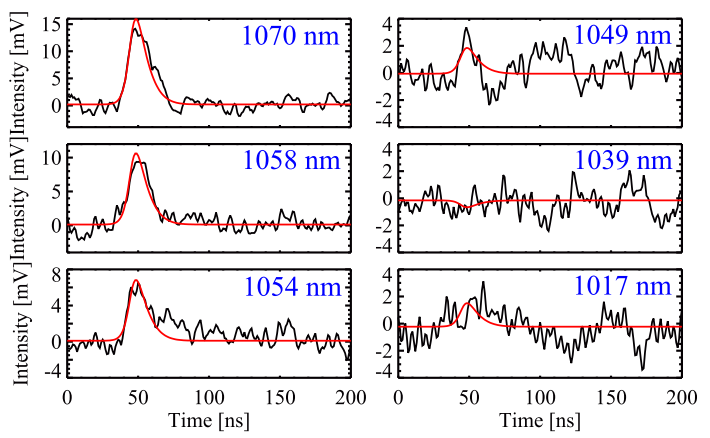

Fig. 2 TS signals and the fittings at $R=342 \mathrm{~mm}$. Blue font values represent the center wavelength of the transmission band of each interference filter in the polychromator.
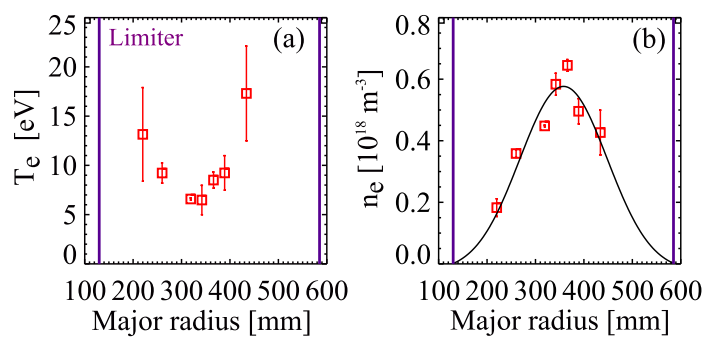

Fig. 3 Profiles for (a) electron temperature $T_{\mathrm{e}}$ and (b) electron density $n_{\mathrm{e}}$ with errors for each are plotted as red open squares. Purple lines represent limiters on both sides. The solid curve in the Fig. (b) shows the Gaussian fit.

integrated density $n_{\mathrm{e}} l$ at the midplane measured by interferometry is approximately $1.3 \times 10^{18} \mathrm{~m}^{-2}(l \sim 0.45 \mathrm{~m})$. Good reproducibility can be seen in the traces of $I_{\mathrm{p}} \mathrm{s}$ and radiation signals. For the TS measurements, we use an $\mathrm{Nd}$ : YAG laser with wavelength $1064 \mathrm{~nm}$, energy $1.6 \mathrm{~J}$, repetition rate $10 \mathrm{~Hz}$, and width $10 \mathrm{~ns}$. The collection system has a scattering angle of $120^{\circ}$ near the plasma center, a solid angle of $0.27 \mathrm{sr}$, fibers with a core diameter of $2 \mathrm{~mm}$, and a numerical aperture of 0.37 . The detection circuit for the polychromator is optimized so that relative errors become small [11]. In the present experiment, $10 \mathrm{TS}$ measurements for 4 spatial points were performed simultaneously, and the $10 \mathrm{TS}$ signals for each spatial point were averaged to attain higher accuracy. Similar measurements were performed for 4 other spatial points, and then profiles with 8 spatial points were obtained. Laser injection time was $t=85 \mathrm{~ms}$, when the plasmas were stable.

Figure 2 shows TS signals at $R=342 \mathrm{~mm}$ obtained by averaging over $10 \mathrm{TS}$ signals and subtracting the background. The signals of 6 wavelength channels in each polychromator were fitted well into a relativistic Maxwellian distribution function. Resultant profiles for $T_{\mathrm{e}}$ and $n_{\mathrm{e}}$ are shown in Fig. 3. Note that one of the spatial points $(R=459 \mathrm{~mm})$ is significantly affected by stray light and the results are not shown in Fig. 3. $T_{\mathrm{e}}$ values in the central region are under $10 \mathrm{eV}$ and the profile is hollow. The $n_{\mathrm{e}}$ profile is like bell-shaped with a peak near $R=342 \mathrm{~mm}$. The relative sensitivity of the $n_{\mathrm{e}}$ measure- ment was obtained by Raman scattering from nitrogen gas. The absolute value of $n_{\mathrm{e}}$ is calibrated by comparing the value of $n_{\mathrm{e}} l$ measured by interferometry with $n_{\mathrm{e}} l$ calculated from the $n_{\mathrm{e}}$ profile measured in an ohmic discharge, where the whole density profile was obtained accurately. The peak density was $n_{\mathrm{e}} \sim 6 \times 10^{17} \mathrm{~m}^{-3}$ and we fitted the data into a Gaussian shape (solid curve in Fig. 3 (b)). The line-integrated density for this shape agrees with $n_{\mathrm{e}} l$ measured by interferometry. In addition, $n_{\mathrm{e}}$ was less than $1 \times 10^{16} \mathrm{~m}^{-3}$ at the outer limiter according to probe measurements. Thus, the fitted Gaussian shape seems to be reasonable, although the density in the peripheral region was not measured.

The shape of the $T_{\mathrm{e}}$ profile obtained is similar to that reported in QUEST, where the plasmas are sustained by ECW power alone [8]. Although ECWs and LHWs are different, the similar hollow $T_{\text {e }}$ profiles may be caused by a common physical phenomenon in RF-sustained ST plasmas.

The central electron pressure for the bulk part of the velocity distribution function obtained by the TS measurement was under $1 \mathrm{~Pa}$. On the other hand, whole plasma pressure including the tail part is calculated from magnetic measurements using an equilibrium reconstruction code EFIT to be around $20 \mathrm{~Pa}$ near the plasma center. Thus, it is suggested that the pressure of fast electrons generated by LHWs is dominant in the equilibrium. In addition, the effective temperature for fast electron estimated from hard X-ray signals is in the order of $10 \mathrm{keV}$, and the ion temperature $T_{\mathrm{i}}$ is determined to be several $\mathrm{eV}$ from spectroscopy [12]. Taking the temperatures and $n_{\mathrm{i}} \simeq n_{\mathrm{e}}$ into account, the density for the fast electrons is in the order of $10^{15} \mathrm{~m}^{-3}$ and the abundance ratio is in the order of $0.1 \%$.

In summary, electron temperature and density profiles in ST plasmas sustained by LHWs have been measured successfully for the first time, helping us understand LHW propagation and absorption in ST plasmas.

This work was supported by Japan Society for the Promotion of Science (JSPS) Grant-in-Aid for Scientific Research No. 21226021 and by the National Institute for Fusion Science (NIFS) Collaborative Research Program Nos. NIFS14KOCR001 and NIFS12KUTR078.

[1] H. Meyer et al., Nucl. Fusion 53, 104008 (2013).

[2] T. Maekawa et al., Nucl. Fusion 45, 1439 (2005).

[3] K. Hanada et al., Plasma Fusion Res. 5, S1007 (2010).

[4] A. Ejiri et al., Nucl. Fusion 46, 709 (2006).

[5] T. Shinya et al., Nucl. Fusion 55, 073003 (2015).

[6] T. Wakatsuki et al., AIP Conf. Proc. 1580, 422 (2014).

[7] V.V. D’yachenko et al., Plasma Phys. Rep. 39, 219 (2013).

[8] T. Yamaguchi et al., Plasma Fusion Res. 8, 1302001 (2013).

[9] M. Yu. Kantor et al., Plasma Phys. Control. Fusion 51, 055002 (2009).

[10] H. Togashi et al., Rev. Sci. Instrum. 85, 056103 (2014).

[11] A. Ejiri et al., Plasma Fusion Res. 5, S2082 (2010).

[12] S. Tsuda et al., Plasma Fusion Res. 10, 1202064 (2015). 